\title{
Editorial comment on: surgical outcomes of two-stage hepatectomy for colorectal liver metastasis: comparison to a benchmark procedure
}

\author{
Zhongkai Wang ${ }^{1}$, Brian K. P. Goh ${ }^{1,2}$ \\ ${ }^{1}$ Department of Hepatopancreatobiliary and Transplant Surgery, Singapore General Hospital, Singapore, Singapore; ${ }^{2}$ Duke-National University of \\ Singapore Medical School, Singapore, Singapore \\ Correspondence to: Brian K. P. Goh, Senior Consultant and Professor. Department of Hepatopancreatobiliary and Transplant Surgery, Singapore \\ General Hospital, 20 College Road, Singapore 169856, Singapore. Email: bsgkp@hotmail.com. \\ Comment on: Mor E, Al-Kurd A, Yaacov AB, et al. Surgical outcomes of two-stage hepatectomy for colorectal liver metastasis: comparison to a \\ benchmark procedure. Hepatobiliary Surg Nutr 2019;8:29-36.
}

Submitted Apr 25, 2021. Accepted for publication May 31, 2021.

doi: $10.21037 / \mathrm{hbsn}-21-166$

View this article at: https://dx.doi.org/10.21037/hbsn-21-166

With advancements in surgical techniques, locoregional therapy and systemic therapy, patients suffering from colorectal cancer with liver metastasis now have numerous therapeutic options for local and systemic disease control. Complete resection of the primary cancer and all metastases remains the primary objective, offering patients the best chance for long-term survival. However, significant challenges arise when patients present with extensive bilobar liver metastases rendering a sufficient post-treatment future liver remnant (FLR) which is critical to avoid hepatic insufficiency, difficult to achieve. This is particularly so in post-chemotherapy patients, due to chemo-induced liver parenchyma damage, whereby a larger FLR is frequently needed to avoid major postoperative complications and even mortality. In these complex clinical scenarios, surgeons need to resort to more advanced strategies to achieve complete tumor clearance. Today, various strategies are available in the armamentarium of the liver surgeon including the combination of liver resection with ablation (1), two-stage hepatectomy (TSH) with portal vein ligation or embolization, associated liver partition and portal vein ligation for staged hepatectomy (ALPPS) (2) and even liver transplantation (3).

In their recent publication, Mor et al. (4) review their results on TSH over a decade, looking at its outcomes in terms of safety, morbidity and mortality. They also compared the outcomes of TSH to standard right hepatectomy (RH) and concluded that TSH was safe in selected patients as evidenced by similar morbidity and mortality rates. Twenty-nine patients underwent TSH, 25 (86.2\%) of whom completed both stages. These patients demonstrated a major complication rate of $17 \%$, and a 90-day mortality rate of $3.4 \%$. Most complications (80\%) were related to the colonic resection, and one patient developed liver failure. Importantly, the TSH cohort demonstrated a 1 -year survival rate of $70.9 \%$, and a respectable median overall survival of 30.2 months.

As reported by the authors (4), considerations such as the number of lesions, comorbidities of the patient and timing of the colonic resection were critical towards a successful TSH. Logically, a larger number of liver lesions leads to more complicated preoperative surgical planning. Of note, the number and of lesions in the planned preserved liver segments, which will contribute directly to the FLR, is critical. Knowledge of other important factors which will determine the final anatomical configuration of the FLR such as location of tumors and their proximity to major vascular and biliary structures is also essential.

It is important to highlight that an important omittance by the authors in their study is that their selection criteria for TSH and detailed information on FLR was not reported. It is critical for the reader to know the FLR cutoff used by the authors to determine the need to perform a TSH versus a single stage resection. Furthermore, is would be essential to know the criteria used such as the minimum FLR needed for a patient to proceed with the second stage 
of resection. It is well-recognized the FLR is an important determinant of morbidity and mortality rates after major liver resection. Over time, different authors have used different criteria to perform a TSH or the need for portal vein embolization with FLR cut-offs ranging from as low as $20 \%$ to $40 \%$. In general, the more aggressive the criteria and the lower the FLR cutoff used, one would expect a higher morbidity and even mortality rate (5).

Over the past few years, with advancements and emerging data on the use of ablative therapies for liver metastasis, ablation has emerged as an important adjunct and even alternative to resection in selected patients. The use of ablation offers potential advantages such as decreased blood loss and improved parenchymal preservation while achieving similar oncological outcomes, particularly in small lesions $(\leq 2 \mathrm{~cm})$ when compared to surgical resection (1). Furthermore, the combination of resection with ablation expands the pool of patients who are eligible for liver resection (1). A note of caution with the use of ablation particularly when used in combination with major liver resections such as TSH, is not to underestimate the final ablation volume when calculating the FLR as this may result in post-operative hepatic insufficiency due to the unaccounted reduction in FLR.

Presently, the adoption of laparoscopic liver resection (LLR) is rapidly increasing in expert liver centers worldwide and has been shown to offer numerous benefits such as shorter length of stay, decreased wound infection, decreased blood loss, decrease post-operative pain and reduced chest infections while offering similar oncological outcomes as the traditional open approach $(6,7)$. In a setting where 2 complex surgical procedures need to be performed in close succession such as TSH, maximizing recovery and minimizing physiological trauma is particularly advantageous. Hence, the use of MIS in TSH has the potential to offer significant improvements in overall outcomes. To date, a number of case series (8) in the literature have reported the feasibility of performing the first stage of the TSH laparoscopically and minimize post-operative pain, the length of stay, earlier commencement of chemotherapy, low morbidity and mortality and frequent progression to second stage surgery with no eventual compromise to the oncological outcome. Furthermore, several authors have also reported the use of MIS for both stages of TSH reporting lower blood loss, shorter length of stay, fewer complications and earlier administration of chemotherapy (8).

It is important to add that in addition to TSH, more surgical options have been introduced over the past decade for the treatment of patients with bilobar liver metastases. Since it was first reported in 2011, ALPPS has emerged as an alternative to TSH and also as a salvage procedure for patients who are unable to proceed to the second stage due to an insufficient FLR. A recent multicenter randomized controlled trial, the LIGRO trial (2) reported a higher resection rate with ALPPS compared to TSH. The study included 97 patients with a standardized FLR of $30 \%$ and reported that ALPPS was superior to TSH in terms of resection rates ( $92 \%$ vs. 52\%), with comparable surgical margins, complications, and short-term mortality (2). Finally, it is also worthwhile to mention the emerging role of liver transplantation for patients with otherwise unresectable bilobar colorectal liver metastasis (3).

\section{Acknowledgments}

Funding: None.

\section{Footnote}

Provenance and Peer Review: This article was commissioned by the editorial office of Hepatobiliary Surgery and Nutrition. The article did not undergo external peer review.

Conflicts of Interest: Both authors have completed the ICMJE uniform disclosure form (available at https://hbsn. amegroups.com/article/view/10.21037/hbsn-21-166/coif). The authors have no conflicts of interest to declare.

Ethical Statement: The authors are accountable for all aspects of the work in ensuring that questions related to the accuracy or integrity of any part of the work are appropriately investigated and resolved.

Open Access Statement: This is an Open Access article distributed in accordance with the Creative Commons Attribution-NonCommercial-NoDerivs 4.0 International License (CC BY-NC-ND 4.0), which permits the noncommercial replication and distribution of the article with the strict proviso that no changes or edits are made and the original work is properly cited (including links to both the formal publication through the relevant DOI and the license). See: https://creativecommons.org/licenses/by-nc-nd/4.0/.

\section{References}

1. Masuda T, Margonis GA, Andreatos N, et al. Combined 
Hepatic Resection and Radio-frequency Ablation for Patients with Colorectal Cancer Liver Metastasis: A Viable Option for Patients with a Large Number of Tumors. Anticancer Res 2018;38:6353-60.

2. Sandström P, Røsok BI, Sparrelid E, et al. ALPPS Improves Resectability Compared With Conventional Two-stage Hepatectomy in Patients With Advanced Colorectal Liver Metastasis: Results From a Scandinavian Multicenter Randomized Controlled Trial (LIGRO Trial). Ann Surg 2018;267:833-40.

3. Dueland S, Syversveen T, Solheim JM, et al. Survival Following Liver Transplantation for Patients With Nonresectable Liver-only Colorectal Metastases. Ann Surg 2020;271:212-8.

4. Mor E, Al-Kurd A, Yaacov AB, et al. Surgical outcomes of two-stage hepatectomy for colorectal liver metastasis: comparison to a benchmark procedure. Hepatobiliary Surg

Cite this article as: Wang Z, Goh BKP. Editorial comment on: surgical outcomes of two-stage hepatectomy for colorectal liver metastasis: comparison to a benchmark procedure. HepatoBiliary Surg Nutr 2021;10(4):570-572. doi: 10.21037/ hbsn-21-166
Nutr 2019;8:29-36.

5. Goh BK. Measured Versus Estimated Total Liver Volume to Preoperatively Assess the Adequacy of Future Liver Remnant: Which Method Should We Use? Ann Surg 2015;262:e72.

6. Syn NL, Kabir T, Koh YX, et al. Survival Advantage of Laparoscopic Versus Open Resection For Colorectal Liver Metastases: A Meta-analysis of Individual Patient Data From Randomized Trials and Propensity-score Matched Studies. Ann Surg 2020;272:253-65.

7. Kabir T, Syn N, Goh BKP. Current status of laparoscopic liver resection for the management of colorectal liver metastases. J Gastrointest Oncol 2020;11:526-39.

8. Okumura S, Goumard C, Gayet B, et al. Laparoscopic versus open two-stage hepatectomy for bilobar colorectal liver metastases: A bi-institutional, propensity scorematched study. Surgery 2019;166:959-66. 